\title{
Características demográficas, de salud, necesidades de cuidado y diagnósticos de enfermería de personas hospitalizadas que sufren trastorno afectivo bipolar*
}

Demographic and Health Characteristics, Care Needs and Nursing Diagnoses in Hospitalized Individuals with Bipolar Affective Disorder

Características demográficas, de saúde, necessidades de cuidado e diagnósticos de enfermagem de pessoal hospitalizado sofrendo transtorno afetivo bipolar

\section{Claudia Patrici Montoya Zapata ${ }^{\text {a }}$}

Universidad Pontificia Bolivariana, Colombia

claudia.montoya@upb.edu.co

ORCID: http://orcid.org/0000-0001-7274-594X

Marjorie Pérez Villa

Universidad Pontificia Bolivariana, Colombia

ORCID: http://orcid.org/0000-0003-1021-7054

Kelly Johana Rendón Morales

Universidad Pontificia Bolivariana, Colombia

ORCID: http://orcid.org/0000-0002-5155-6688

Alexandro Antonio Quintero Arenas

Universidad Pontificia Bolivariana, Colombia

ORCID: http://orcid.org/0000-0003-1318-2950

Isabel Cristina Urrego Febrys

Universidad Pontificia Bolivariana, Colombia

ORCID: http://orcid.org/0000-0002-8819-2013

Matilde Ligia Álvarez Gómez

Universidad Pontificia Bolivariana, Colombia

ORCID: http://orcid.org/0000-0003-2093-898X
DOI: https://doi.org/10.11144/Javeriana.ie21-1.cdsn Redalyc: http://www.redalyc.org/articulo.oa? id $=145257605002$

Fecha de recepción: 27 Febrero 2017 Fecha de aprobación: 23 Noviembre 2017

\section{Resumen:}

Introducción: el trastorno afectivo bipolar $(\mathrm{TAB})$ es una enfermedad mental crónica más frecuente en mujeres que en hombres. La prevalencia en el mundo es del $1 \%$ y en Colombia es del 1,2\%. Objetivo: Describir las características demográficas, de salud, necesidades de cuidado y diagnóstico de enfermería en pacientes que ingresan a hospitalización con un diagnóstico de TAB a la Clínica Psiquiátrica Nuestra Señora del Sagrado Corazón. Método: Estudio descriptivo retrospectivo. Se seleccionaron 158 historias clínicas entre enero y junio de 2015, La información se recolectó de las notas de ingreso del equipo terapéutico, y para las necesidades de cuidado se utilizaron los patrones funcionales de Marjory Gordon. Resultados: el 55,7\% eran mujeres, con edades entre 19 y 82 años, el 71,5\% eran residentes de Medellín, el 47,5\% eran solteros, el 19\% tenían estudios secundarios. El 66,5\% presentó sintomatología propia del TAB en el momento del ingreso; el diagnóstico CIE-10 más frecuente fue TAB-episodio maniaco, presente con síntomas psicóticos. Las necesidades encontradas, según los patrones de Marjory Gordon, fueron: falta de introspección, presencia de insomnio, alucinaciones y autoagresión. Para estas necesidades los diagnósticos de enfermería fueron: deterioro de la toma de decisiones independiente, trastorno del patrón del sueño y riesgo de violencia autodirigida. Conclusiones: La información demográfica y de salud es similar a las características reportadas por otras investigaciones. Tanto las necesidades de cuidado y los diagnósticos de enfermería son congruentes con la incapacidad mental que impiden un adecuado autocuidado.

Palabras clave: trastorno bipolar, atención de enfermería, diagnóstico de enfermería.

\footnotetext{
a Autor de correspondencia: claudia.montoya@upb.edu.co
} 


\begin{abstract}
:
Introduction: Bipolar Affective Disorder (BAD) is a chronic mental illness more frequently found in woman than in men. The prevalence over the world is $1 \%$ and in Colombia is $1.2 \%$. Objective: To describe the demographic and health characteristics, care needs and nursing diagnoses in patients hospitalized due to a BAD diagnosis in the mental-health clinic Clínica Psiquiátrica Nuestra Señora del Sagrado Corazón. Method: This is a retrospective descriptive study. One hundred and fifty-eight medical records were selected between January and June 2015. Information was gathered from the admission notes by the therapeutic team; the functional patterns by Marjory Gordon were used to examine the care needs based on the data. Results: In the sample, $55.7 \%$ were women from 19 to 82 years old; $71.5 \%$ of the sample lived in Medellín; $47.5 \%$ were single individuals; $19 \%$ had completed the high school. A $66.5 \%$ showed symptoms typical to the BAD at the time of admission; under the CIE-10 diagnosis, the BAD maniac episode was the most frequent and included psychotic symptoms. According to the patterns by Marjory Gordon, the nursing needs found in the sample were: lack of introspection, sleeplessness, hallucinations, and selfharming. Regarding these needs, the nursing diagnoses were: deterioration in the independent decision-making; disorder of the sleep pattern and self-harming risk. Conclusions: The demographic and health information is similar to the typical characteristics reported in other researches. Both the care needs and nursing diagnoses are consistent with the mental inability, which hampers an appropriate self-care.
\end{abstract}

Keywords: bipolar disorder, nursing care, nursing diagnosis.

\title{
Resumo:
}

Introdução: $\mathrm{O}$ transtorno afetivo bipolar $(\mathrm{TAB})$ é uma doença mental crônica mais frequente em mulheres do que em homens. A prevalência no mundo é de $1 \%$ e na Colômbia é de 1,2\%. Objetivo: Descrever as características demográficas, de saúde, necessidades de cuidado e diagnóstico de enfermagem em pacientes internos com diagnóstico de TAB na Clínica Psiquiátrica Nuestra Señora del Sagrado Corazón. Método: Estudo descritivo retrospectivo. Selecionaram-se 158 relatórios clínicos entre janeiro e junho de 2015. A informação foi coletada das anotações de admissão da equipe terapêutica, e para as necessidades de cuidado foram utilizados padrões funcionais de Marjory Gordon. Resultados: 55,7\% foram mulheres, com idades entre 19 e 82 anos, $71,5 \%$ foram residentes em Medellín, 47,5\% solteiros, 19\% tinham ensino médio. 66,5\% apresentou sintomatologia própria do TAB no momento do ingresso; o diagnóstico CIE-10 mais frequente foi TAB-episódio maníaco, presente com sintomas psicóticos. As necessidades encontradas, de acordo com os padrões de Marjory Gordon, foram: falta de introspeção, presença de insônia, alucinações e autoagressão. Para tais necessidades os diagnósticos de enfermagem foram: deterioração da tomada de decisões independente, transtorno do padrão de sonho e risco de violência autodirigida. Conclusões: As informações demográficas e de saúde são semelhantes às caraterísticas relatadas por outras pesquisas. Tanto as necessidades de cuidado quanto os diagnósticos de enfermagem são congruentes com a incapacidade mental que impede um adequado autocuidado.

Palavras-chave: transtorno bipolar, atenção de enfermagem, diagnóstico de enfermagem.

\section{Introducción}

El trastorno afectivo bipolar (TAB) es una enfermedad mental crónica frecuente. Se caracteriza por episodios depresivos y maniacos mixtos o hipomaniacos que a menudo están separados por un intervalo asintomático $(1,2)$. Según criterios diagnósticos del Manual diagnóstico y estadístico de los trastornos mentales (DSM), esta enfermedad se clasifica en: bipolar I (episodios maniacos e hipomaniacos y episodio de depresión), bipolar II (uno o más episodios depresivos y, por lo menos, un episodio hipomaniaco actual o pasado), trastorno ciclotímico (fluctuaciones del ánimo con numerosos periodos de síntomas hipomaniacos, que se alternan con otros de síntomas depresivos) $(3,4)$.

En el mundo, la prevalencia de TAB es del 1\% (5) y en Estados Unidos se reportan prevalencias de trastorno bipolar I de 0,6\% (4), y en América Latina la prevalencia de episodio maniaco fue de: 1,2\% en Colombia; $0,9 \%$ en Chile; $1,3 \%$ en ciudad de México y $0,5 \%$ en Costa Rica (6). En la ciudad de Medellín el trastorno bipolar tiene una prevalencia anual en población general de $0,7 \%$ (7).

La edad de inicio de la enfermedad puede ser variada: desde una edad temprana, con un promedio de 17,7 años; intermedia, de 23,9 años, y tardía, de 48,2 años (8). Es más frecuente en mujeres que en hombres. En Medellín, un estudio sobre agregación familiar y características de pacientes con TAB, halló que el 70\% eran mujeres (9). En Costa Rica, otra investigación sobre caracterización clínica de estas personas encontró 
que el 57,7\% eran de este mismo sexo (10). En la población general de Colombia existen 2,6 mujeres con el diagnóstico por cada hombre $(11,12)$.

Con frecuencia, las personas con TAB tienen otros trastornos comórbidos, y dentro de los más frecuentes están los trastornos por uso de sustancias (TUS) $(13,14,15,16)$. En mujeres adolescentes también se han informado trastornos de la conducta alimentaria (17), al igual que la hipertensión arterial (30\%) (12), asma y concentraciones elevadas de lípidos (19,2\%) y enfermedades de la tiroides (14\%) (18).

Para realizar el cuidado, enfermería utiliza para la valoración e identificación de las necesidades de salud los patrones funcionales de Marjory Gordon (19,20,21). Al revisar la literatura científica en cuidados de enfermería de personas que sufren $\mathrm{TAB}$, se ha encontrado que las necesidades de cuidados más frecuentes fueron la agitación, la agresión verbal, la actividad física excesiva, los problemas relacionados con la medicación, la carencia de introspección, el irrespeto en los límites de los otros, el comportamiento caótico, las reacciones extremas, los problemas con las relaciones, entre otros (22,23).

Con la identificación de las necesidades de cuidado se proponen diagnósticos de enfermería (23). Entre ellos, los que más se formulan a las personas hospitalizadas con TAB son desempeño ineficaz de rol, incumplimiento secundario a falta de conciencia de la enfermedad y afrontamiento familiar comprometido $(23,24,25,26)$.

El objetivo de esta investigación fue describir las características sociales y de salud, las necesidades de cuidado y los diagnósticos de enfermería de un grupo de personas que sufrían TAB y que ingresaron al servicio de hospitalización en un centro de salud mental de la ciudad de Medellín.

\section{Método}

Esta investigación se planteó como un estudio descriptivo retrospectivo. La muestra fue no probabilística con muestreo a conveniencia, constituida por 430 historias clínicas de pacientes hospitalizados, de ambos sexos, mayores de edad con diagnóstico de TAB. El criterio de inclusión era tener registro clínico completo; de estos, 158 pacientes cumplían con este.

Para recolectar la información se construyeron dos instrumentos basados en los objetivos del estudio y la operacionalización de las variables. El primero contenía información social (edad, estrato, escolaridad y ocupación) y de salud (motivo de consulta, diagnóstico médico, inicio de la enfermedad, comorbilidad mental, antecedentes personales de enfermedad física y antecedentes familiares de enfermedad mental).

El segundo estaba constituido por una lista de revisión con las necesidades de cuidado según los patrones funcionales de salud de Marjory Gordon (percepción de la salud, nutricional/metabólico, eliminación, actividad/ejercicio, sueño/descanso, cognitivo/perceptivo, autopercepción/autoconcepto, rol/relaciones, sexualidad/reproducción, adaptación/tolerancia al estrés, valores/creencias).

Para formular los diagnósticos de enfermería, se partió de los patrones funcionales de Marjory Gordon. A partir de estos se abordaron las necesidades de cuidado de los pacientes y se interrelacionaron con las etiquetas de la Asociación Norteamericana de Diagnósticos de Enfermería (NANDA), teniendo en cuenta que cumplieran con las características definitorias para el diagnóstico.

La información se tomó de la nota de ingreso realizada por médicos, enfermeras y psicólogos, y esta se consignó en una base de base de datos en el programa Excel. Posteriormente, se pasó a programa SPSS version 22 y se realizó un análisis univariado calculando frecuencias absolutas y relativas para las variables cualitativas y promedios y medianas para las variables cuantitativas.

Esta investigación fue aprobada por la institución donde se recolectó la información y el Comité de Ética de Investigación de la Escuela de Ciencias de la Salud. Se consideró sin riesgo para la salud según Resolución 8430 de 1993, emanada del Ministerio de Salud de la República de Colombia (27). 


\section{Resultados}

Se revisaron las notas de ingreso de los pacientes con diagnóstico de TAB, de los cuales 158 cumplían con los criterios de inclusión.

Entre las variables demográficas más relevantes se encontró que la mediana de la edad fue de 48 años. El $55,7 \%$ (88) eran mujeres, el 57,6\% (91) no tenía pareja, el 65,8\% (104) pertenecía al régimen contributivo, el 35,4\% (56) tenía algún tipo de ingreso y el 84,2\% (133) residía en zona urbana (tabla 1).

TABLA1

Características sociodemográficas de los pacientes con trastorno afectivo bipolar $(\mathrm{n}=158)$

\begin{tabular}{|c|c|c|c|}
\hline \multicolumn{2}{|c|}{ Característica } & \multirow{2}{*}{$\begin{array}{r}\mathbf{n}=\mathbf{1 5 8} \\
48 \\
\end{array}$} & \multirow{2}{*}{\begin{tabular}{|c|}
$\%$ \\
$35-5$
\end{tabular}} \\
\hline Edad & \begin{tabular}{|l} 
Mediana \\
(percentil 25-75)
\end{tabular} & & \\
\hline \multirow{2}{*}{ Sexo } & Masculino & 70 & 44,3 \\
\hline & Femenino & 88 & 55,7 \\
\hline \multirow{5}{*}{ Estado civil } & \begin{tabular}{|l|} 
Soltero \\
\end{tabular} & 75 & 47,5 \\
\hline & Casado & 45 & 28,5 \\
\hline & Unión libre & 22 & 13,9 \\
\hline & Divorciado & 7 & 4,4 \\
\hline & Viudo & 9 & 5,7 \\
\hline \multirow{3}{*}{ Seguridad social } & $\begin{array}{l}\text { Régimen } \\
\text { subsidiado }\end{array}$ & 48 & 30,4 \\
\hline & $\begin{array}{l}\text { Régimen } \\
\text { contributivo }\end{array}$ & 104 & 65,8 \\
\hline & Sin dato & 6 & 3,8 \\
\hline \multirow{2}{*}{ Tener pareja } & Con pareja & 67 & 42,4 \\
\hline & Sin pareja & 91 & 57,6 \\
\hline \multirow{3}{*}{ Ocupación } & Sin ingresos & 31 & 19,6 \\
\hline & Con ingresos & 56 & 35,4 \\
\hline & Sin información & 71 & 44,9 \\
\hline \multirow{2}{*}{ Lugar de residencia } & Medellín & 113 & 71,5 \\
\hline & Otro municipio & 45 & 28,5 \\
\hline \multirow{6}{*}{ Estrato socioeconómico } & Bajo bajo & 32 & 20,3 \\
\hline & Bajo & 35 & 22,2 \\
\hline & Medio bajo & 63 & 39,9 \\
\hline & Medio & 2 & 1,3 \\
\hline & Medio alto & 2 & 1,3 \\
\hline & Sin información & 24 & 15,2 \\
\hline \multirow{10}{*}{ CIE-10 } & F310 & 1 & 0,6 \\
\hline & F311 & 2 & 1,3 \\
\hline & F312 & 56 & 35,4 \\
\hline & F313 & 3 & 1,9 \\
\hline & F314 & 26 & 16,5 \\
\hline & F315 & 7 & 4,4 \\
\hline & F316 & 13 & 8,2 \\
\hline & F317 & 5 & 3,2 \\
\hline & F318 & 11 & 7,0 \\
\hline & F319 & 34 & 21,5 \\
\hline
\end{tabular}

Fuente: elaboración propia. 
Según la décima versión de la Clasificación Internacional de las Enfermedades (CIE-10), el principal diagnóstico de los pacientes con $\mathrm{TAB}$ fue trastorno afectivo bipolar episodio maniaco presente con síntomas sicóticos [F312], con un 35,4\% (56); seguido de trastorno afectivo bipolar episodio depresivo grave presente sin síntomas psicóticos [F314], con un 16,5\% (26).

El principal motivo de consulta de ingreso al servicio de hospitalización fue la presencia de síntomas relacionados con TAB, con un $66,5 \%$ (105). En cuanto a los antecedentes de los pacientes, se encontró que el grupo de enfermedades que predominaban eran las endocrinas y las metabólicas, con un 21,5\% (34). La principal comorbilidad mental fue el TUS, con un 27,8\% (44), y el antecedente familiar mental para los trastornos afectivos fue del $17,7 \%$ (28) (tabla 2).

TABLA 2

Características de salud de los pacientes con trastorno afectivo bipolar $(\mathrm{n}=158)$

\begin{tabular}{|c|c|c|c|}
\hline \multicolumn{2}{|r|}{ Característica } & $\mathrm{n}=158$ & $\%$ \\
\hline \multirow{4}{*}{$\begin{array}{l}\text { Motivo de } \\
\text { consulta }\end{array}$} & Remitido & 33 & 20,9 \\
\hline & Sin adherencia al tratamiento & 4 & 2,5 \\
\hline & Sintomático & 105 & 66,5 \\
\hline & Sin información & 16 & 10,1 \\
\hline \multirow{12}{*}{$\begin{array}{l}\text { Antecedentes } \\
\text { personales }\end{array}$} & Enfermedad cardiovascular & 20 & 12,7 \\
\hline & Enfermedad pulmonar & 1 & 0,6 \\
\hline & Enfermedad gastrointestinal & 2 & 1,3 \\
\hline & Enfermedad endocrina-metabólica & 34 & 21,5 \\
\hline & Enfermedad renal-genitourinaria & 1 & 0,6 \\
\hline & $\begin{array}{l}\text { Enfermedad músculo-esquelética y } \\
\text { de tejido conectivo }\end{array}$ & 4 & 2,5 \\
\hline & Enfermedad infecciosa & 5 & 3,2 \\
\hline & Enfermedad neurológica & 12 & 7,6 \\
\hline & $\begin{array}{l}\text { Enfermedad oncológica- } \\
\text { hematológica }\end{array}$ & 2 & 1,3 \\
\hline & Enfermedad dermatológica & 2 & 1,3 \\
\hline & Enfermedad ginecológica & 2 & 1,3 \\
\hline & Sin información & 73 & 46,2 \\
\hline \multirow{7}{*}{$\begin{array}{l}\text { Comorbilidad } \\
\text { mental }\end{array}$} & Esquizofrenia & 10 & 6,3 \\
\hline & $\begin{array}{lll}\begin{array}{l}\text { Trastorno } \\
\text { neurológico }\end{array} & \text { del } & \text { desarrollo } \\
\end{array}$ & 4 & 2,5 \\
\hline & Trastornos afectivos & 9 & 5,7 \\
\hline & Trastornos de ansiedad & 3 & 1,9 \\
\hline & Trastorno por uso de sustancias & 44 & 27,8 \\
\hline & Otros & 10 & 6,3 \\
\hline & Sin información & 78 & 49,4 \\
\hline \multirow{7}{*}{$\begin{array}{l}\text { Antecedentes } \\
\text { familiares de } \\
\text { comorbilidad } \\
\text { mental }\end{array}$} & Esquizofrenia & 11 & 7,0 \\
\hline & $\begin{array}{lll}\begin{array}{l}\text { Trastorno } \\
\text { neurológico }\end{array} & \text { del } & \text { desarrollo } \\
\end{array}$ & 1 & 0,6 \\
\hline & Trastornos afectivos & 28 & 17,7 \\
\hline & Trastornos de ansiedad & 1 & 0,6 \\
\hline & Trastorno por uso de sustancias & 4 & 2,5 \\
\hline & Otros & 18 & 11,4 \\
\hline & Sin información & 95 & 60,1 \\
\hline \multirow{5}{*}{$\begin{array}{l}\text { Consumo de } \\
\text { sustancias } \\
\text { psicoactivas }\end{array}$} & Alcohol & 33 & 20,9 \\
\hline & Cigarrillo & 26 & 16,5 \\
\hline & Cocaina & 12 & 7,6 \\
\hline & Uso crónico de benzodiacepinas & 1 & 0,6 \\
\hline & Sin información & 86 & 54,4 \\
\hline
\end{tabular}

Fuente: elaboración propia. 
Con la lista de chequeo elaborada con los patrones funcionales de Marjory Gordon, se obtuvieron los principales síntomas psiquiátricos y las necesidades de cuidado de los pacientes con TAB. Estas necesidades se interrelacionaron con las etiquetas diagnósticas de la NANDA y se utilizó la taxonomía II de la NANDA 2015-2017 para extraer los diagnósticos de enfermería (tabla 3).

TABLA 3

Diagnósticos enfermeros más comunes para las intervenciones del trastorno afectivo bipolar

\begin{tabular}{|c|c|c|c|c|c|}
\hline \multirow{2}{*}{$\begin{array}{l}\text { Patrón } \\
\text { funcional de } \\
\text { Gordon }\end{array}$} & \multirow{2}{*}{$\begin{array}{l}\text { Sintomatología } \\
\text { psiquiátrica o } \\
\text { necesidad de } \\
\text { cuidado }\end{array}$} & \multirow{2}{*}{$\mathbf{n}$} & \multirow{2}{*}{$\%$} & \multicolumn{2}{|c|}{ Diagnósticos enfermeros } \\
\hline & & & & Dominio NANDA & $\begin{array}{c}\text { Etiqueta } \\
\text { diagnóstica }\end{array}$ \\
\hline \multirow[b]{2}{*}{$\begin{array}{l}\text { Percepción de } \\
\text { la salud }\end{array}$} & \multirow[b]{2}{*}{$\begin{array}{l}\text { Carencia de } \\
\text { introspección }\end{array}$} & \multirow[b]{2}{*}{123} & \multirow[b]{2}{*}{77,8} & $\begin{array}{l}\text { 1. Promoción de la salud } \\
\text { Clase 2: Gestión de la salud }\end{array}$ & $\begin{array}{l}(00078) \text { Gestión } \\
\text { ineficaz de la } \\
\text { salud }\end{array}$ \\
\hline & & & & $\begin{array}{l}\text { 10. Principios vitales } \\
\text { Clase } 3: \text { Congruencia entre } \\
\text { valores/creencias/acciones }\end{array}$ & $\begin{array}{l}(00242) \\
\text { Deterioro de la } \\
\text { toma de } \\
\text { decisiones } \\
\text { independiente } \\
\end{array}$ \\
\hline Sueño-descanso & $\begin{array}{l}\text { Presencia de } \\
\text { insomnio }\end{array}$ & 91 & 57,6 & $\begin{array}{l}\text { 4. Actividad y reposo } \\
\text { Clase 2: Actividad/ejercicio }\end{array}$ & $\begin{array}{l}(00198) \\
\text { Trastomo del } \\
\text { patrón del sueño }\end{array}$ \\
\hline $\begin{array}{l}\text { Cognitivo- } \\
\text { perceptivo }\end{array}$ & $\begin{array}{l}\text { Alucinaciones o } \\
\text { ilusiones }\end{array}$ & 69 & 43,7 & $\begin{array}{l}\text { 5. Percepción/cognición } \\
\text { Clase 4: Cognición }\end{array}$ & $\begin{array}{l}\text { (00251)Control } \\
\text { emocional } \\
\text { inestable }\end{array}$ \\
\hline $\begin{array}{l}\text { Autopercepción } \\
\text {-autoconcepto }\end{array}$ & $\begin{array}{l}\text { Megalomanía o } \\
\text { grandiosidad }\end{array}$ & 40 & 25,3 & $\begin{array}{l}\text { 9. Afrontamiento/tolerancia } \\
\text { Clase 2: Respuestas de } \\
\text { a frontamiento }\end{array}$ & $\begin{array}{l}(00071) \\
\text { Afrontamiento } \\
\text { defensivo }\end{array}$ \\
\hline Rol-relaciones & Autoagresión & 59 & 37,3 & $\begin{array}{l}\text { 11. Seguridad/protección } \\
\text { Clase } 3 \text { : Violencia }\end{array}$ & $\begin{array}{l}\text { (00140) Riesgo } \\
\text { de violencia } \\
\text { autodirigida }\end{array}$ \\
\hline
\end{tabular}

Fuente: elaboración propia.

\section{Discusión}

Con el desarrollo de esta investigación se conocieron las características demográficas, de salud, necesidades de cuidado y diagnósticos de enfermería según patrones funcionales de Marjory Gordon (19,20,23).

Algunas de las condiciones demográficas de esta población — como la edad, el sexo y el estado civil— son similares a otras investigaciones realizadas en Medellín (9). Aunque el trastorno bipolar más frecuente en la población general es el TAB I (5), entre los datos recolectados de las historias clínicas de los pacientes se encontró que el más frecuente fue el afectivo bipolar episodio maniaco presente con síntomas sicóticos. Esto se puede explicar, porque no todas las personas con $\mathrm{TAB}$ requieren hospitalización (1); otro motivo puede ser porque los pacientes maniacos psicóticos son disfuncionales desde el punto de vista social y están con ideas delirantes y alucinaciones, lo que dificulta el manejo en el hogar, por lo que ameritan hospitalización (28).

En la literatura científica, al igual que lo que se halló en esta investigación, es frecuente encontrar relación entre TAB y enfermedades metabólicas y cardiovasculares (12,29). En este estudio el antecedente personal más destacado fue la hipertensión arterial, seguido de trastornos tiroideos, lo que coincide con otros reportes (18).

La comorbilidad mental más frecuente encontrada fueron los TUS, con $27 \%$. Esta frecuencia fue menor a las informada por otros investigadores que van del $40 \%$ (30) al $76 \%$ (16). El menor porcentaje encontrado en este estudio pudo deberse a la separación entre consumo simple y el abuso y dependencia, y que muchos de los 
reportados como consumos simples pudieron haber sido TUS. Al igual que en otros estudios, las sustancias psicoactivas más consumidas fueron el alcohol y el tabaco $(14,31)$.

La carencia de introspección fue la necesidad de cuidado más encontrada. Esta misma fue registrada en otra investigación cualitativa realizada en personas que sufren esta enfermedad (22). La introspección hace parte del patrón de percepción de la salud y alude al reconocimiento que se tiene de la enfermedad. La carencia de ella es tanto un síntoma psiquiátrico como una necesidad de cuidado de enfermería; y entre los diagnósticos de enfermería que corresponden a esta necesidad se encuentra la gestión ineficaz de la salud y el deterioro de la toma de decisiones. Estos no coinciden con el síntoma mental, ya que el sujeto no es consciente de su condición, este no es voluntario y hace parte en unión con otros del trastorno mental (23).

La segunda necesidad en orden de frecuencia fue el insomnio, que pertenece al patrón funcional sueñodescanso. Es muy frecuente hallarla en personas que sufren otros trastornos mentales; al igual que la anterior, es un síntoma mental que puede ser intervenido a través de cuidados de enfermería. Esta necesidad ha sido registrada en la literatura propia del cuidado de personas con TAB (24,26,32), y es también un diagnóstico de enfermería (33).

En casi la mitad de las personas estudiadas se reportaron alteraciones perceptivas e ideas delirantes. Se sabe que ambas son síntomas propios del TAB con síntomas psicóticos (34); pero, a su vez, son necesidades de cuidado. Ellas hicieron parte de los diagnósticos de enfermería, cuya denominación era deterioro de la interpretación del entorno (35).

El diagnóstico de enfermería para la necesidad "alucinaciones" es control emocional inestable, y aunque este es un síntoma no voluntario, es una alteración perceptiva no emocional (23). El cuidado de enfermería está caracterizado por su enfoque holístico, cuidar a la persona desde los aspectos biológicos, sociales, psicológicos, culturales y espirituales. La enfermera para realizar el cuidado tiene en cuenta el proceso de atención de enfermería e identifica en sus pacientes las necesidades de cuidado de forma individual (valoración), detectando en ellos alteraciones reales y potenciales que llevan al planteamiento de los diagnósticos enfermeros, a efectos de mejorar la situación de los pacientes (planificación y ejecución) y al final evaluar los resultados. Las necesidades de cuidado, junto con los diagnósticos de enfermería de los pacientes con $\mathrm{TAB}$, permitirán planear un cuidado con calidad, para mejorar el manejo actual y prevenir complicaciones adicionales a su patología.

\section{Conclusiones}

Los resultados de esta investigación muestran que la población estudiada tiene características demográficas y de salud similares a las características reportadas en otras investigaciones. Con base en los hallazgos, se encontró que los diagnósticos de enfermería — trastorno del patrón del sueño, vagabundeo y desequilibrio nutricional - están de acuerdo con las necesidades de cuidado y son congruentes con la incapacidad mental que impiden un adecuado autocuidado.

\section{Agradecimientos}

A la Clínica Psiquiátrica Nuestra Señora del Sagrado Corazón, por apoyarnos y permitirnos realizar esta investigación.

\section{Referencias}

1. Yepes L. Trastorno bipolar. En: Toro R, Yépez L PC, editores. Fundamentos de medicina: psiquiatría. 5a ed. Medellín: CIB; 2013. p. 195-207. 
2. Townsend MC. Trastornos de estado de ánimo. En: Fundamentos de enfermería en psiquiatría. 4a ed. Madrid: McGraw Hill; 2008. p. 332-73.

3. González Parra D, González de María V, Leal Sánchez C, Sánchez Iglesias S. Enfermedad bipolar. Med (Baltimore) [internet]. 2007;9(85):5461-8, Disponible en: https://www.dialnet.unirioja.es/servlet/extart?codigo=235979 4.

4. American Psychiatric Association. Diagnostic and statistical manual of mental disorders (DSM-5). Washington: APA; 2013.

5. Nieto J, Calvo R. Patrones de comorbilidad en pacientes psiquiátricos ambulatorios. Rev Colomb Psiquiatr. 2001;30(1):21-37.

6. Vicente B, Rioseco P, Saldivia S, Kohn R, Torres S. Prevalencia de trastornos psiquiátricos en Latinoamérica: revisión crítica. Rev Colomb Psiquiatr. 2005;34(4):506-14.

7. Tores de Galvis Y, editora. Primer Estudio Poblacional de Salud Mental Medellín, 2011-2012, Medellín: Universidad CES, Universidad de Harvard, Secretaría de Salud de Medellín; 2012.

8. Pedraza RS, Losada JR, Jaramillo LE. Edad de inicio como marcador de subtipos de enfermedad maniacodepresiva. Rev Colomb Psiquiatr. 2012;41(3):576-87. https://www.doi.org/10.1016/S0034-7450(14)60030-5

9. Ricardo J, Calle C, Palacio J. Efecto de la agregación familiar en la caracterización clínica del trastorno afectivo bipolar tipo I: variables en pacientes de población antioqueña. Rev Colomb Psiquiatr. 2003;32(2):145-60.

10. Raventós JCH. Caracterización clínica de familias costarricenses con trastorno afectivo bipolar. Acta Médica Costarric. 2014;56(4):167-73.

11. Medina ME, Borges GL, Muñoz CBC, Blanco Jaimes J, Fleiz Bautista C, Villatoro Velázquez J, et al. Prevalencia de trastornos mentales y uso de servicios: resultados de la encuesta nacional de epidemiología. Salud Ment [internet]. 2003;26(4):1-16. Disponible en: https://www.redalyc.org/articulo.oa?id=58242601.

12. Moreno LJA, Giraldo CÁ, Castrillón JJC, Gil MM, Jiménez MPM, Borja NO, et al. Relación entre trastorno afectivo bipolar, depresión, ansiedad, estrés y valores de presión arterial en población de la clínica San Juan de Dios. Arch Med (Manizales) [internet]. 2015;15:281-90. Disponible en: https://www.revistasum.umanizales.e du.co/ojs/index.php/archivosmedicina/article/view/1027/1464.

13. Casas M, Franco MD, Goikolea JM, Roncero C, Szerman N, Bipolar T. Trastorno bipolar asociado al uso de sustancias adictivas (patología dual): revisión sistemática de la evidencia científica y consenso entre profesionales expertos. Área. 2008;36(6):350-61.

14. Pérez de Heredia JL, González Pinto A, Ramírez M, Imaz A, Ruiz J. Abuso y dependencia de sustancias en el trastorno bipolar. Trastor Adict [internet]. 2001;3(S2):1-64. Disponible en: https://www.elsevier.es/es-revistatrastornos-adictivos-182-articulo-abuso-dependencia-sustancias-el-trastorno-13013506.

15. López-Ortiz C, Roncero C, Miquel L, Casas M. Fumar en las psicosis afectivas: revisión sobre el consumo de nicotina en el trastorno bipolar y esquizoafectivo. Adicciones. 2011;23(1):65-75.

16. Torres Y, Posada J, Barreño J, Berbesí D. Trastornos por abuso y dependencia de sustancias en población colombiana: su prevalencia y comorbilidad en otros trastornos mentales seleccionados. Rev Colomb Psiquiatr. 2010;39:14-35. https://www.doi.org/10.1016/S0034-7450(14)60265-1

17. Zubrzycki A. Trastorno bipolar. Salud Ciencia. 2011;18:290.

18. Forty L, Ulanova A, Jones L, Jones I, Gordon-Smith K, Fraser C, et al. Comorbid medical illness in bipolar disorder. Br J Psychiatr. 2014;205(6):465-72. https://www.doi.org/10.1192/bjp.bp.114.152249.

19. Patrones funcionales de Marjory Gordon [internet]. (Citado 2017 ene 21). Disponible en: http:// www.ome.es/04_01_desa.cfm?id=391.

20. Álvarez Suárez JL, Castillo Arévalo F del, Fernández Fidalgo D, Muñoz Meléndez M. Manual de valoración de patrones funcionales. Gijón: Dirección de Enfermería de Atención Primaria; 2010.

21. Martínez-López MA, Pérez-Constantino M, Montelongo-Meneses PP. Proceso de atención de enfermería a una lactante con neumonía basado en patrones funcionales de Marjory Gordon. Enferm Univ. 2014;11(1):36-43. 
22. Daggenvoorde T, Geerling B, Goossens PJ. A qualitative study of nursing care for hospitalized patients with acute mania. Arch Psychiatr Nurs. 2015;29:186-91. https://www.doi.org/10.1016/j.apnu.2015.02.003.

23. NANDA International. Diagnósticos enfermeros: definiciones y clasificación, 2015-2017.3a ed. Madrid: Elsevier; 2016.

24. Muñoz MJZ, González FJM. Paciente con trastorno bipolar: proceso de enfermería. ENE. Rev Enferm [internet]. 2012;6(2):81-8. Disponible en: https://www.ene-enfermeria.org/ojs/index.php/ENE/article/view/20.

25. Herrero Villanueva JA, Serrano M, Parra MD. Planes de cuidados al paciente con depresión y trastorno bipolar. Cat-barcelona.com [internet]. 2016. Disponible en: https://www.cat-barcelona.com/pdf/biblioteca/trastorno -bipolar/4_-_planes_de_cuidados_al_paciente_con_depresion_y_trastorno_bipolar.pdf.

26. Álvarez M. Cuidado de personas que sufren trastornos mentales. En: Enfermería en psiquiatría y salud mental. Medellín: Universidad Pontificia Bolivariana; 2013. p. 35-94.

27. Resolución 8430 del 4 de octubre de 1993, por la cual se establecen las normas científicas, técnicas y administrativas para la investigación en salud

28. Ministerio de Sanidad, Servicios Sociales e Igualdad. Guía de práctica clínica sobre trastorno bipolar. Madrid: Ministerio; 2012.

29. Jaramillo CL, Mejía AC, Velásquez AH, Restrepo Palacio TF, Zuluaga JO. Síndrome metabólico y trastorno afectivo bipolar: una revisión de la literatura. Rev Colomb Psiquiatr [internet]. 2013;42(3):283-91. Disponible en: https://www.sciencedirect.com/science/article/pii/S0034745013700210.

30. Sánchez-Moreno J, Martínez-Arán A, Vieta E. Comorbilidad en el trastorno bipolar. Rev Psiquiatr Fac Med. 2005;32(2):77-88.

31. Pardo Cely EE, Fierro M, Ibáñez Pinilla M. Prevalencia y factores asociados a la no adherencia en el tratamiento farmacológico de mantenimiento en adultos con trastorno afectivo bipolar. Rev Colomb Psiquiatr. 2011;40:85-98.

32. Townsend MC. Trastorno bipolar. En: Fundamentos de enfermería psiquiátrica. 4a ed. Mexico: McGraw-Hill; 2008. p. 252-373.

33. Herdman TH, Kamitsuru S, editores. NANDA International Nursing Diagnoses: definitions and classification 2012-2014. p. 31-561.

34. Angst J. The bipolar spectrum. Br J Psychiatry. 2007;190(1922):189-91. https://doi.org/10.1192/ bjp.bp.106.030957.

35. Dochterman JM, Jones DA, Hahn K. Unifying nursing languages: The harmonization of NANDA, NIC, and NOC. Int J Nurs Terminol Classif. 2004;15(2):34.

\section{Notas}

*Artículo original de investigación

\section{Licencia Creative Commons CC BY 4.0}

Cómo citar: Montoya Zapata CP, Pérez Villa M, Rendón Morales KJ, Quintero Arenas AA, Urrego Febrys IC, Álvarez Gómez ML. Características demográficas, de salud, necesidades de cuidado y diagnósticos de enfermería de personas hospitalizadas que sufren trastorno afectivo bipolar. Investig Enferm Imagen Desarr. 2019;21(1). https://www.doi.org/10.11144/Javeriana.ie21-1.cdsn 